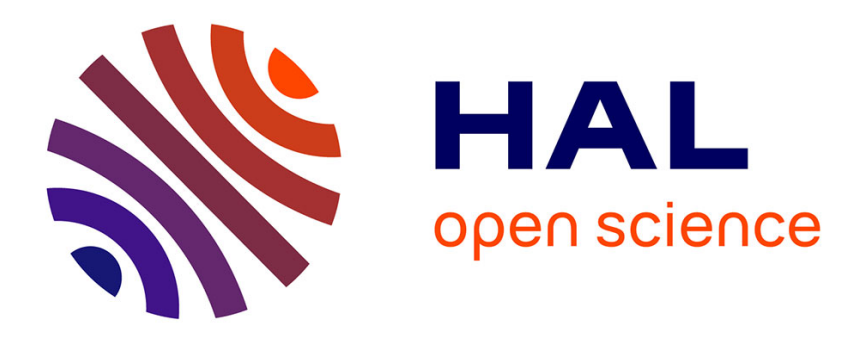

\title{
Propagation des ondes dans les guides à plasma
}

Philippe Leprince

\section{To cite this version:}

Philippe Leprince. Propagation des ondes dans les guides à plasma. Revue de Physique Appliquée, 1967, 2 (4), pp.239-244. 10.1051/rphysap:0196700204023900 . jpa-00242798

\section{HAL Id: jpa-00242798 https://hal.science/jpa-00242798}

Submitted on 1 Jan 1967

HAL is a multi-disciplinary open access archive for the deposit and dissemination of scientific research documents, whether they are published or not. The documents may come from teaching and research institutions in France or abroad, or from public or private research centers.
L'archive ouverte pluridisciplinaire HAL, est destinée au dépôt et à la diffusion de documents scientifiques de niveau recherche, publiés ou non, émanant des établissements d'enseignement et de recherche français ou étrangers, des laboratoires publics ou privés. 


\title{
PROPAGATION DES ONDES DANS LES GUIDES A PLASMA (1)
}

\author{
Par Philippe LEPRINGE, \\ Laboratoire de Physique des Plasmas, Orsay.
}

\begin{abstract}
Résumé. - Après un rappel des études faites sur la propagation des ondes le long d'une colonne de plasma sans champ magnétique, on compare théoriquement diverses structures de guide qui peuvent toutes propager deux types de modes : les modes de plasma qui n'existent qu'en présence du plasma et les modes de guide qui existent sans plasma. La présence d'une onde inverse et l'existence de vitesse de phase supérieure à la vitesse de la lumière sont étudiées pour les modes de plasma. Dans la dernière partie, les couplages entre deux types de modes sont mis en évidence lorsque la fréquence du plasma est supérieure à la fréquence de coupure des modes de guide sans plasma.
\end{abstract}

Abstract. - Having recalled studies on wave propagation along an unmagnetised plasma column, for various guide structures two different types of mode are studied : plasma modes, that is modes existing only with plasma, and waveguide modes that exist without plasma.

The presence of a backward wave and the existence of phase velocity greater than the speed of light is studied for the plasma mode. In the last section, coupling between those two different modes is evidenced when the plasma frequency is greater than the cutoff frequency of the plasma less waveguide mode.

Introduction. - La propagation des ondes le long d'une colonne de plasma a fait l'objet ces dernières années de nombreuses études théoriques et expérimentales. Cet intérêt est dû, en particulier, à la possibilité d'utiliser les plasmas dans des dispositifs actifs ou passifs en hyperfréquence. La première étude, faite par A. W. Trivelpiece et R. W. Gould, a montré la possibilité de propager une onde inverse le long d'une

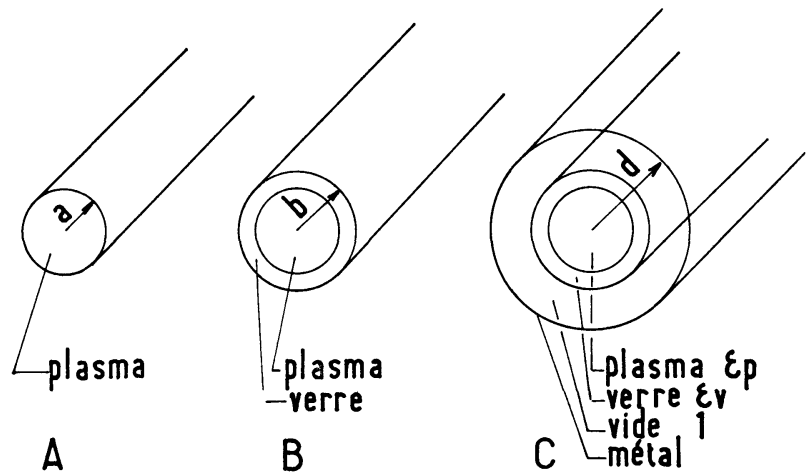

FIG. 1. - Structure des guides à plasma.

colonne de plasma en présence ou non d'un champ magnétique extérieur; depuis cette date, différents auteurs ont étudié l'une des structures de la figure 1. Dans le présent article, nous présentons une synthèse

(1) Cette étude a été partiellement financée par un contrat de la Délégation Générale à la Recherche Scientifique et Technique. de ces résultats en indiquant les différences entre les trois structures et en discutant les propriétés des modes : modes de guide qui existent sans la présence du plasma, modes de plasma qui n'existent qu'en présence de ce dernier. Dans les deux paragraphes suivants, nous nous bornerons à l'étude des modes dipolaires $m=1$ ( $m$ désignant l'indice azimutal lié au facteur $\mathrm{e}^{j m \varphi}$ dans les expressions des champs) en insistant spécialement sur l'influence des divers paramètres sur la présence d'une onde inverse et sur la présence d'un couplage entre le mode de plasma et les modes de guide.

1. Equation de dispersion. - Avant de faire l'étude de la propagation, il faut connaître la constante diélectrique du plasma; pour la calculer, nous devons faire un certain nombre d'hypothèses :

- Le plasma est neutre mais, comme nous n'étudierons que la propagation des hautes fréquences, les ions seront considérés comme immobiles;

- Les collisions entre les électrons et les neutres ou les ions n'auront aucune influence sur la propagation; cela suppose que les fréquences de collisions $\nu_{\text {en }}$ (électrons-neutres) et $\nu_{\mathrm{ei}}$ (électrons-ions) sont petites par rapport à la fréquence de l'onde :

$$
\frac{\nu_{\mathrm{en}}}{\omega} \quad \text { et } \quad \frac{\nu_{\mathrm{ei}}}{\omega} \ll 1 \text {. }
$$

- Nous négligerons les effets dus à la température électronique; cette hypothèse aura deux conséquences: les courbes de dispersion ne seront valables que dans 
le domaine où la vitesse de phase de l'onde est supérieure à la vitesse d'agitation thermique; de plus, cette théorie ne pourra pas expliquer toutes les courbes expérimentales : certains modes de propagation ne seront pas décrits par l'équation de dispersion que nous obtiendrons [8], [9];

- Nous considérons que le plasma est homogène et que les variations radiale et longitudinale de densité n'influent pas sur la propagation. On a montré que cette hypothèse, fausse a priori, permet néanmoins d'obtenir avec une bonne précision les courbes de dispersion [10].

Compte tenu de ces hypothèses, la constante diélectrique du plasma s'écrit :

$$
\varepsilon_{0} \varepsilon_{\mathrm{p}}=\varepsilon_{0}\left(1-\frac{\omega_{\mathrm{p}}^{2}}{\omega^{2}}\right)
$$

où

$$
\omega_{\mathrm{p}}^{2}=n e^{2} / m_{\mathrm{e}} \varepsilon_{0} .
$$

Les guides à plasma que nous allons étudier consistent en des structures composites à deux ou trois diélectriques dont l'un (le plasma) a une permittivité soit négative $\left(\omega<\omega_{\mathfrak{p}}\right)$, soit positive $\left(\omega>\omega_{\mathfrak{p}}\right)$.

L'équation de dispersion s'obtient en résolvant les équations de Maxwell dans les divers milieux et en appliquant les conditions aux limites. Ce calcul ne présente pas de difficultés : il conduit à une équation de dispersion qu'il est commode d'écrire, pour discuter les propriétés des divers modes, sous la forme d'un déterminant [14]. Nous nous contenterons ici d'en rappeler une des particularités : les divers composants des champs sont de la forme :

$$
E=E_{0} J_{m}(K r) \mathrm{e}^{j m \varphi} \mathrm{e}^{\gamma z} \mathrm{e}^{j \omega t}
$$

avec

$$
\begin{aligned}
& K^{2}=\gamma^{2}-k_{0}^{2} \varepsilon \\
& k_{0}=\omega / c
\end{aligned}
$$

$\varepsilon$ : constante diélectrique du milieu.

Mais, contrairement au cas des guides classiques à un seul diélectrique, on ne peut en général, dans les structures composites de la figure 1 , séparer les modes $E$ et les modes $H$. Il existe des termes de couplage qui ne s'annulent que dans deux cas :

$-m=0:$ les modes à symétrie axiale sont soit $E$, soit $H$;

$-\gamma=0:$ aux fréquences de coupure de tous les modes, l'onde est soit $E$, soit $H$; cela nous permettra par la suite de distinguer deux sortes de modes : les modes $E H$ qui sont $E$ à la coupure et les modes $H E$ qui sont $H$ à la coupure.

Pour une structure donnée (structure $\mathrm{C}$ ), la figure 2 donne l'allure des courbes de dispersion pour les
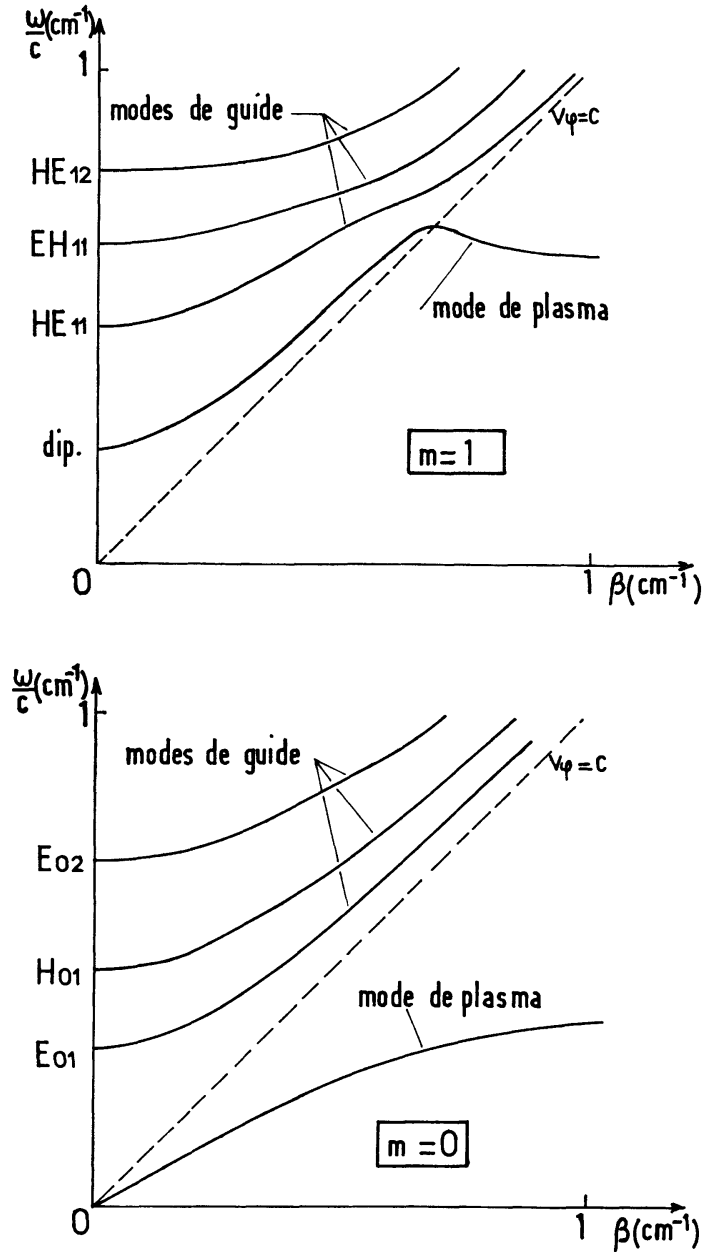

Fig. 2. - Courbes de dispersion ( $m=0$ et $m=1)$ pour une structure de guide $\mathrm{C}$ :

$a=0,4 \mathrm{~cm} ; b=0,5 \mathrm{~cm} ; d=8 \mathrm{~cm}$;

$\varepsilon_{\mathrm{v}}=5 ; \quad \frac{\omega_{\mathrm{p}}}{c}=1,4 \mathrm{~cm}^{-1}$.

modes à symétrie axiale et pour les modes dipolaires. On voit apparaître deux catégories de modes :

- Des modes de guide qui existent sans la présence du plasma;

- Des modes supplémentaires qui sont dus uniquement à la présence du plasma et qui existent même sans guide métallique entourant le plasma : ils sont appelés soit modes de surface, car, du moins pour les faibles vitesses de phase, l'énergie de l'onde est localisée à la surface du plasma, soit modes de plasma; nous conserverons cette dernière appellation qui nous semble la plus correcte et la plus générale.

2. Modes de plasma. - Pour bien comprendre l'influence des divers milieux qui peuvent entourer la colonne de plasma, nous avons tracé ( fig. 3 ) les courbes de dispersion des deux premiers modes $(m=0, m=1)$ pour les deux premières structures $\mathrm{A}$ et $\mathrm{B}$ de la figure 1 . 

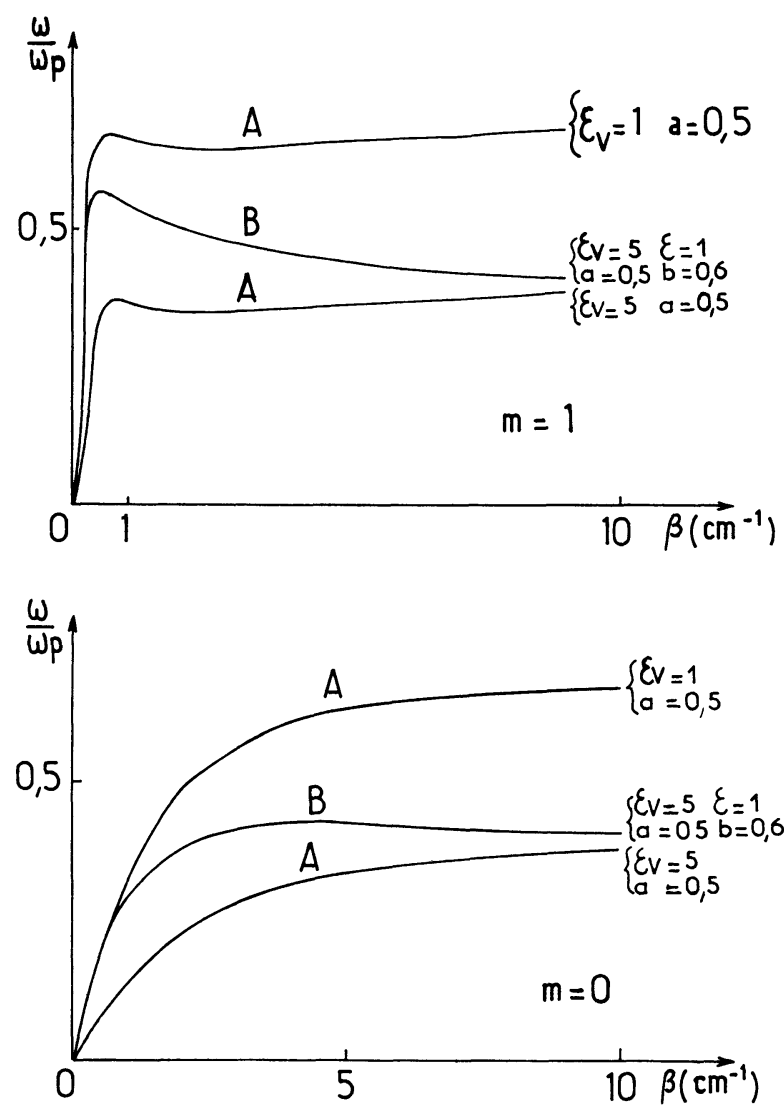

FIG. 3. - Courbes de dispersion des modes de plasma ( $m=0$ et $m=1$ ) pour les structures A et B : $a=0,5 \mathrm{~cm} ; b=0,6 \mathrm{~cm} ; \varepsilon_{\mathrm{v}}=1$ ou 5 .

2.1. DANS LE GAS D'Un Guide A DeUX MILIEUX (structure A, le plasma et un diélectrique), la figure permet de dire :

- Que la fréquence de résonance $(\beta \rightarrow \infty)$ dépend de la permittivité du milieu qui entoure le plasma :

$$
\omega_{\text {res }}=\omega_{\mathrm{p}} / \sqrt{1+\varepsilon_{\mathrm{v}}}
$$

où $\varepsilon_{\mathrm{v}}$ est la permittivité relative;

- Que le mode à symétrie axiale ne présente qu'une onde directe, tandis que le mode dipolaire possède une onde inverse dont la bande passante est d'autant plus faible que la fréquence est élevée.

2.2. DANS LE GAS D'UN GUIDE A TROIS MILIEUX (structure B, plasma, verre, vide), les conclusions sont les suivantes :

- La courbe de dispersion est entièrement comprise entre celles des structures à deux milieux : plasma-verre et plasma-vide;

- La fréquence de résonance dépend uniquement de la permittivité du verre;

- Une onde inverse existe pour les deux modes, et en particulier pour le mode dipolaire; la bande passante de l'onde inverse est augmentée par la présence du verre.

REVUE DE PHYSIQUe APPLIQUÉE. - T. 2. No 4. DÉCEMBRE 1967.
Cette étude permet de comprendre l'influence de la constante diélectrique du verre et de son épaisseur : pour les grandes valeurs de $\beta(\lambda \rightarrow 0)$, seul le verre joue un rôle; le champ haute fréquence décroît radialement très rapidement à partir de la limite plasma-verre. Lorsque $\beta$ diminue, cette influence sera d'autant plus importante que l'épaisseur du verre sera grande par rapport à la longueur d'onde dans ce milieu. Pour les faibles valeurs de $\beta$, le verre n'a plus aucune importance (le champ décroît très lentement).

Nous allons maintenant donner quelques détails complémentaires pour le mode dipolaire en étudiant d'une part l'influence de la densité ainsi que l'influence de la présence d'un guide métallique entourant le plasma (structure $\mathrm{G}$ ).

3. Mode dipolaire. - De nombreuses études de ce mode ont été publiées [6], [11] à [13], mais il nous semble intéressant de faire ici deux remarques sur les conditions d'obtention d'une onde inverse ainsi que sur l'existence d'une onde rapide lorsque la colonne se trouve dans un guide métallique.

3.1. ONDE INVERSE. - L'approximation quasi statique qui avait permis de mettre en évidence l'existence de l'onde inverse [6], [11] ne pouvait en aucun cas donner des indications sur la largeur de bande en fonction de la fréquence. L'étude exacte, que nous avons faite [14], permet d'écrire que, pour une structure de dimensions données, plus la fréquence est élevée, plus la largeur de bande est faible; l'onde inverse disparaît même pour les grandes valeurs de la fréquence plasma ( fig. 4). Pour une fréquence donnée pour avoir une onde inverse, il faut choisir une colonne

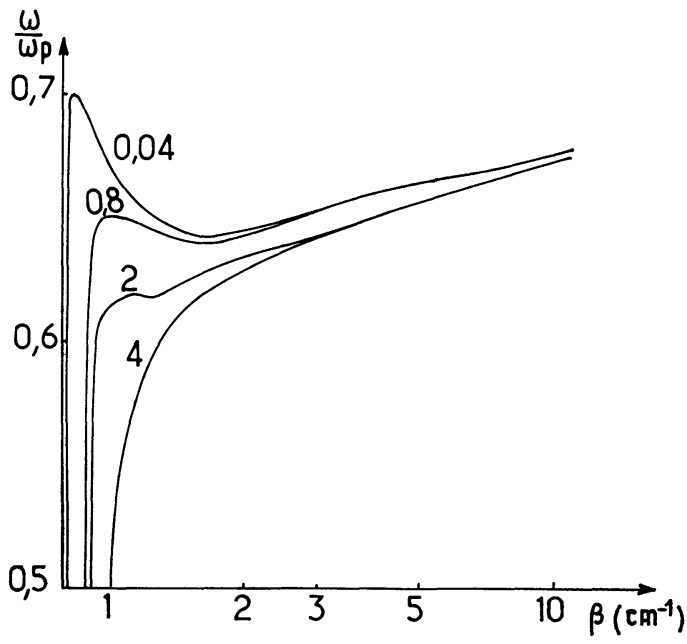

FIG. 4. - Influence de la densité sur l'existence d'une onde inverse pour le mode de plasma $m=1$ (structure B) :

$$
\begin{aligned}
& a=0,5 \mathrm{~cm} ; b=0,6 \mathrm{~cm} ; \\
& \varepsilon_{\mathrm{v}}=5 \text { paramètre }\left(\frac{\omega_{\mathrm{p}}}{c}\right)^{2}\left(\mathrm{~cm}^{-2}\right) .
\end{aligned}
$$


de plasma aussi petite que possible, entourée d'un diélectrique de constante diélectrique élevée et de faible épaisseur.

3.2. Onde RAPIDE. - L'influence d'un guide métallique entourant le plasma (structure $\mathrm{C}$ ) est en général très peu importante pour le domaine des ondes lentes, sauf lorsque le diamètre du guide tend vers celui de la colonne; à titre d'exemple, la figure 5 donne une série de courbes de dispersion pour une

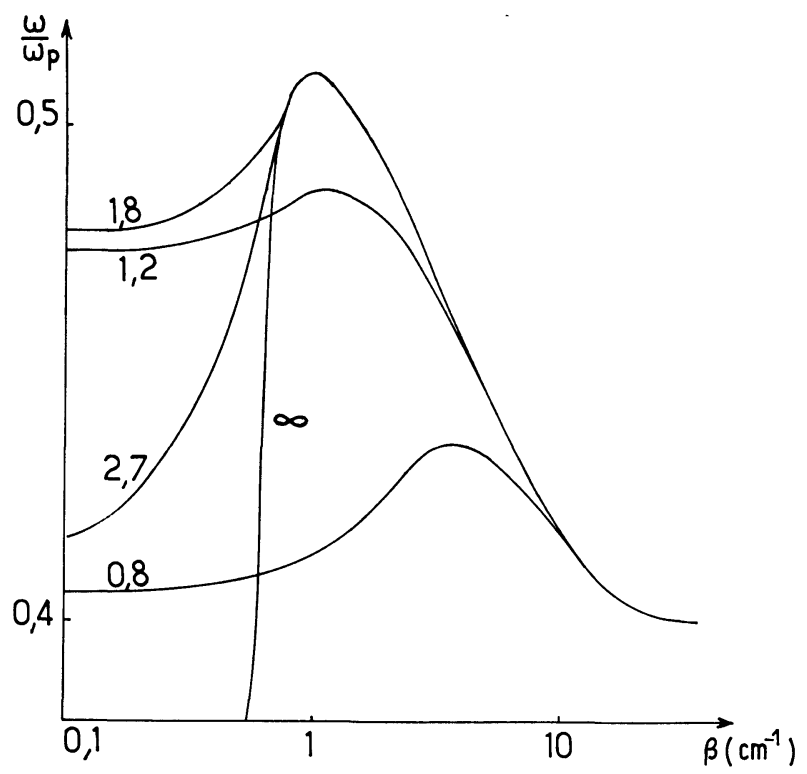

FIG. 5. - Influence du diamètre du guide métallique sur la courbe de dispersion du mode de plasma $m=1$ (structure C) :

$$
\begin{aligned}
& a=0,5 \mathrm{~cm} ; b=0,6 \mathrm{~cm} ; \\
& \varepsilon_{\mathrm{v}}=5 ; \quad\left(\frac{\omega_{\mathrm{p}}}{c}\right)=1,4 \mathrm{~cm}^{-1} ; \text { paramètre } d(\mathrm{~cm}) .
\end{aligned}
$$

colonne de plasma de densité donnée pour divers diamètres du guide métallique.

Contrairement au mode à symétrie axiale (dont la vitesse de phase est toujours inférieure à celle de la lumière), le mode dipolaire possède une onde rapide ( fig. 2). L'étude de la fréquence de coupure est assez complexe [14], mais nous pourrons la résumer ainsi : pour une colonne donnée, la fréquence de coupure croît lorsque le diamètre du guide diminue (toujours inférieure à la fréquence de coupure du mode $\left.\mathrm{TM}_{11}\right)$, puis décrô̂t ensuite; la fréquence de coupure maximum est de l'ordre de $\omega_{\mathrm{p}} / 2$. L'existence d'une onde rapide permet des couplages avec les modes de guide que nous allons étudier maintenant.

4. Modes de guide $(m=1)$. - Les modes à symétrie axiale ne présentent pas de particularités et ont fait l'objet de nombreuses études; aussi nous ne parlerons ici que des modes $m=1$ dont les courbes de dispersion peuvent être très modifiées par la présence du plasma. Deux cas se présentent :

- Lorsque les fréquences de propagation du mode de plasma et des modes de guide sont très distinctes, ces derniers ne sont que faiblement perturbés et il n'existe aucune déformation notable des courbes de dispersion ( fig. 6);

- Lorsque les fréquences de propagation sont du même ordre de grandeur, il y a couplage entre les modes. C'est ce cas que nous allons étudier maintenant.

4.1. Les gourbes de dispersion. - La figure 6 montre l'évolution des courbes de dispersion lorsque la densité de plasma croît, on remarque qu'il y a couplage entre les modes de plasma et le premier mode de guide, et par suite toutes les courbes de dispersion se décalent. Pour mieux montrer la zone de couplage, nous l'avons tracée sur la même fi-
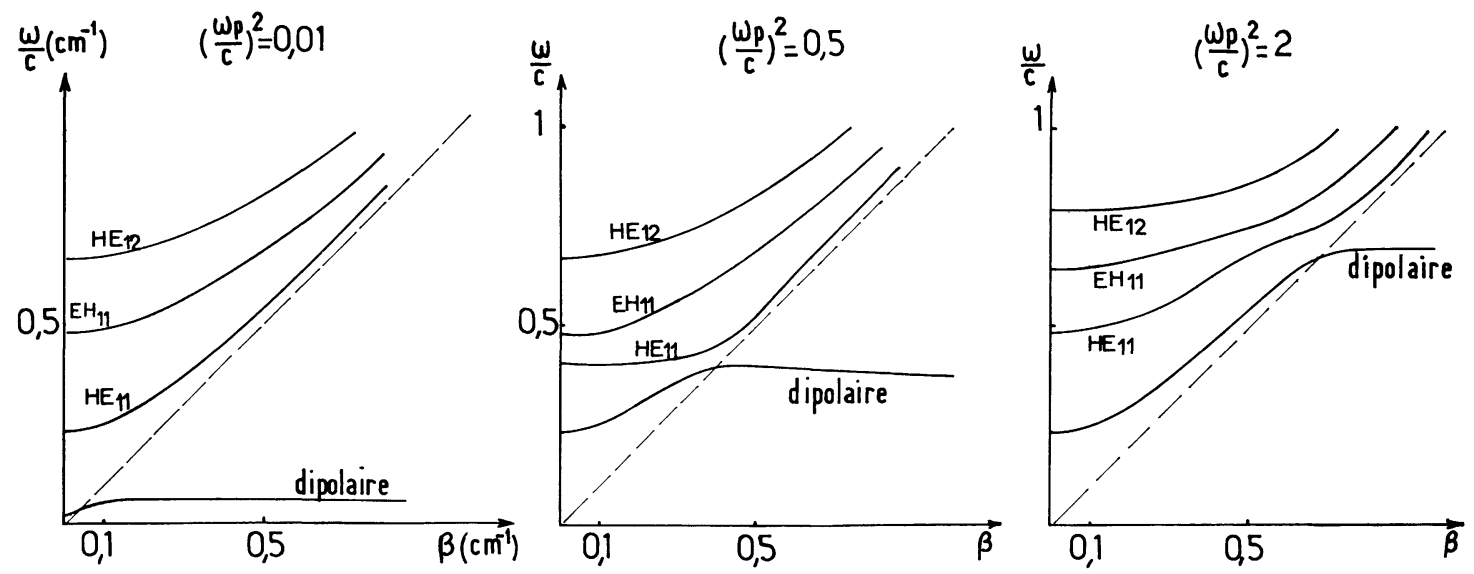

FIG. 6. - Courbe de dispersion des modes dipolaires pour diverses valeurs de densité du plasma :

$$
a=0,4 \mathrm{~cm} ; b=0,5 \mathrm{~cm} ; d=8 \mathrm{~cm} ; \varepsilon_{\mathrm{v}}=5 \text {. }
$$


gure (fig. 7) pour une même structure mais pour plusieurs valeurs de la fréquence plasma. Contrairement à ce que l'on pourrait penser, le couplage est d'autant plus

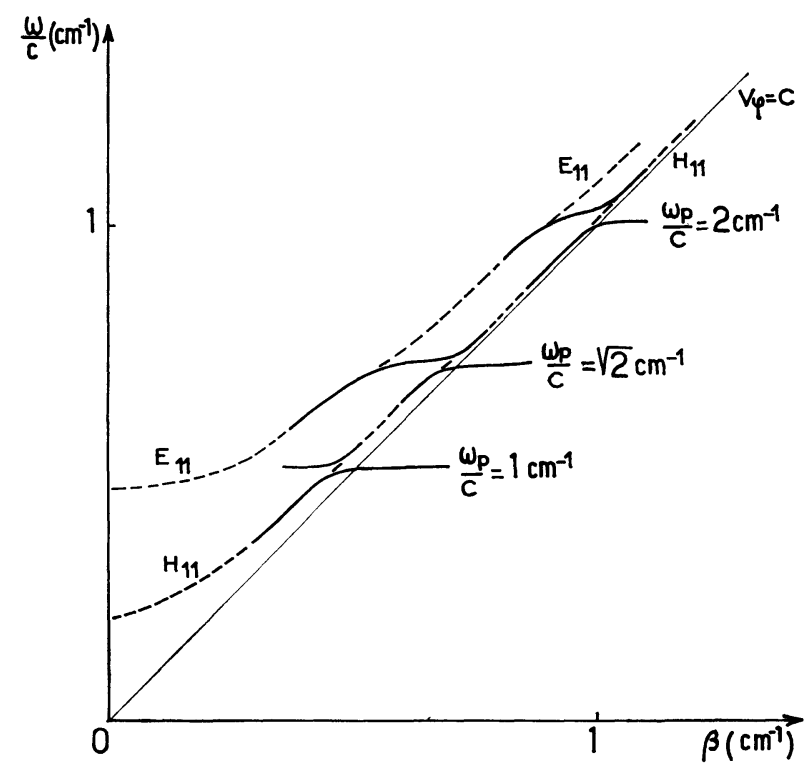

Fig. 7. - Couplage entre les modes de guide et le mode de plasma :

$$
a=0,4 \mathrm{~cm} ; b=0,5 \mathrm{~cm} ; d=8 \mathrm{~cm} ; \varepsilon_{\mathrm{v}}=5 .
$$

net que le diamètre du plasma est petit; en effet, ce qui compte, ce n'est pas le volume du plasma par rapport à celui du guide (comme pour l'étude du décalage en fréquence de la résonance d'une cavité contenant un plasma), mais le rapport entre la fréquence de propagation des modes de guide sans

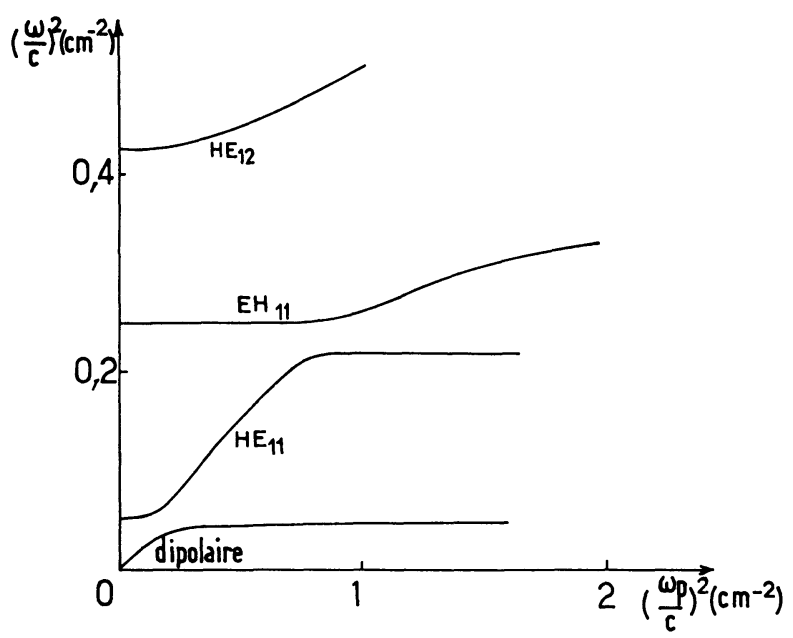

Fig. 8. - Fréquence de coupure des modes $m=1$ en fonction de la fréquence plasma :

$$
a=1 \mathrm{~cm} ; b=1,2 \mathrm{~cm} ; d=8 \mathrm{~cm} ; \varepsilon_{\mathrm{v}}=5 .
$$

plasma et celle du mode dipolaire (qui est d'autant plus élevée que la colonne de plasma a un faible diamètre, tous les autres paramètres étant identiques).

Cela nous permet d'écrire qu'un guide à plasma peut être considéré comme le couplage de deux systèmes qui ont leurs courbes de dispersion propres : le guide et la colonne de plasma.

4.2. Les fréquences de Goupure $(\beta=0)$. - Pour bien montrer l'évolution des courbes de dispersion, il est intéressant d'étudier celle de la fréquence de coupure en fonction de la densité du plasma (fig. 8); on remarque que, lorsque cette dernière croît, la fréquence de coupure du mode dipolaire tend vers celle du mode $E_{11}$ sans plasma, celle du mode $E H_{11}$ vers celle du mode $H_{11}$ sans plasma, et ainsi de suite. Une remarque identique peut être faite pour la résonance d'une cavité de longueur $L(\beta \neq 0)$ contenant un plasma [14].

Conclusion. - Pour faire cette étude, nous avons dû faire quelques hypothèses qui ne sont pas tout à fait valables, et pour conclure nous allons parler de l'une d'entre elles qui nous paraît importante. Si l'on ne tient pas compte de la vitesse thermique des électrons, non seulement les courbes de dispersion ne sont exactes que pour des vitesses de phase nettement supérieures à la vitesse thermique, mais également une série de modes, dont la vitesse de phase peut être supérieure à celle de la lumière, ne sont pas décrits. Par suite, il existe des couplages entre ces modes et

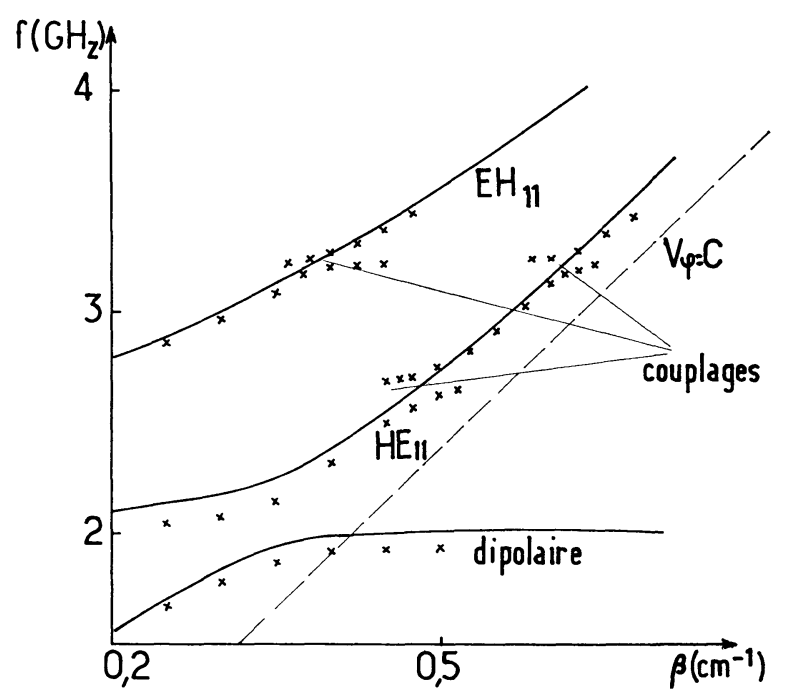

Frg. 9. - Courbe de dispersion expérimentale pour les modes $m=1$ (en haut, à droite, lire : $V_{\varphi}=c$ ) :

— courbes théoriques,

$\times \times \times \times$ points expérimentaux,

$a=0,4 \mathrm{~cm} ; b=0,5 \mathrm{~cm} ; d=8 \mathrm{~cm} ; \varepsilon_{\mathrm{v}} \simeq 4,8$. 
ceux du guide, et les divers diagrammes de Brillouin que nous avons tracés ne sont pas complets. A titre d'exemple, nous donnons un diagramme ( fig. 9), obtenu expérimentalement pour les modes $m=1$, qui montre avec précision de nouveaux couplages entre les modes.
Remerciements. - Je remercie M. le Professeur Delcroix pour les conseils qu'il m'a prodigués tout au long de ce travail et Mme Claudette Leprince qui a programmé les diverses équations de dispersion.

Manuscrit reçu le 7 juin 1967.

\section{BIBLIOGRAPHIE}

[1] Trivelpiece (A. V.) et Gould (R. W.), J. Appl. Physics, 1959, 30, 1784.

[2] Schumann (W. O.), Z. Angew. Physics, 1960, 12, 10.

[3] BEC (M.) et EVERHART (T. E.), J. of Elec. and Cont., 1962, 13, 185.

[4] Ar.FREDSson (L.), Trans. Roy. Inst. Technol., 1964, 13, 233.

[5] Leprince (P.) et Pommier (J.), Proc. IEE, 1966, 113, 588.

[6] Carlilie (R. N.), J. Appl. Physics, 1964, 35, 1384.

[7] Clarricoats (P. J. B.) et al., Proc. IEE, 1966, 113, 755.
[8] Diament (P.) et al., J. Appl. Physics, 1966, 37, 1771

[9] Crawford (F. W.) et TATARONIS (J. A.), J. Electron Control, 19, 557.

[10] Pommier (J.), Étude du mode dipolaire, Thèse $3^{\text {e }}$ cycle, 1966.

[11] Akao (Y.) et IdA (Y.), J. Appl. Physics, 1964, 35, 2565.

[12] Granatstein (V. L.) et SchiesingeR (S. P.), $J$. Appl. Physics, 1965, 36, 3503.

[13] LEPRINCE (P.), Phenomena in Ionized Plasmas, Beograd, 1965.

[14] LEPRINCE (P.), Rapport interne LP 63, Lab. de Phys. des Plasmas, Orsay. 